\title{
ON THE RADIUS-EDGE CONDITION IN THE CONTROL VOLUME METHOD*
}

\author{
GARY L. MILLER ${ }^{\dagger}$, DAFNA TALMOR ${ }^{\ddagger}$, SHANG-HUA TENG ${ }^{\S}$, AND \\ NOEL WALKINGTON $₫$
}

\begin{abstract}
In this paper we show that the control volume algorithm for the solution of Poisson's equation in three dimensions will converge even if the mesh contains a class of very flat tetrahedra (slivers). These tetrahedra are characterized by the fact that they have modest ratios of diameter to shortest edge, but large circumscribing to inscribed sphere radius ratios, and therefore may have poor interpolation properties. Elimination of slivers is a notoriously difficult problem for automatic mesh generation algorithms. We also show that a discrete Poincaré inequality will continue to hold in the presence of slivers.
\end{abstract}

Key words. mesh generation, slivers, control volume method

AMS subject classifications. $65 \mathrm{M} 12,52 \mathrm{C} 22$

PII. S0036142996311854

1. Introduction. We consider covolume approximations of Poisson's equation on a three-dimensional domain. It is shown that the control volume algorithm will converge on Delaunay meshes that contain both well proportioned tetrahedra and certain degenerate tetrahedra called "slivers," that is, flat tetrahedra whose vertices are approximately equispaced around the equator of a sphere (see Figure 1). This result was rather unexpected, since the classical finite element algorithm may fail to converge when such degenerate elements are present.

Classical finite element theory shows that the finite element solution is the best approximation of the solution in the $H_{0}^{1}(\Omega)$ norm, ${ }^{1}$ so clearly the control volume algorithm can't converge in this norm if the finite element solution doesn't. Instead we show that the control volume scheme converges in a discrete $H_{0}^{1}(\Omega)$ norm $\left(\|\cdot\|_{W}\right.$ below), and like the finite element algorithm, the control volume algorithm gives the best projection with respect to this norm. Numerical experiments indicate that the finite element method may fail to converge in either norm. We note that in two dimensions the two norms coincide, so this dichotomy is truly a three-dimensional phenomenon. Since the discrete norm used with the control volume algorithm changes with the mesh, it is natural to ask if convergence can be established in any of the standard norms. We answer this in the affirmative by establishing a discrete Poincaré

*Received by the editors November 11, 1996; accepted for publication (in revised form) August 24, 1998; published electronically September 17, 1999.

http://www.siam.org/journals/sinum/36-6/31185.html

†School of Computer Science, Carnegie Mellon University, Pittsburgh, PA 15213 (glmiller@ theory.cs.cmu.edu). The work of this author was supported in part by NSF grant CCR-9505472.

${ }^{\ddagger}$ School of Computer Science, Carnegie Mellon University, Pittsburgh, PA 15213 (tdafna@ cs.cmu.edu).

$\S$ Department of Computer Science, University of Minnesota, Minneapolis, MN 55455. The work of this author was supported in part by an NSF CAREER award (CCR-9502540) and an Alfred P. Sloan Research Fellowship.

『Department of Mathematics, Carnegie Mellon University, Pittsburgh, PA 15213 (noelw@ cmu.edu.). The work of this author was supported in part by National Science Foundation grant DMS-9203406. This work was also supported by the Army Research Office and NSF through the Center for Nonlinear Analysis.

$$
{ }^{1}\|u\|_{H_{0}^{1}(\Omega)}^{2}=\int_{\Omega}|\nabla u|^{2} \text {. }
$$




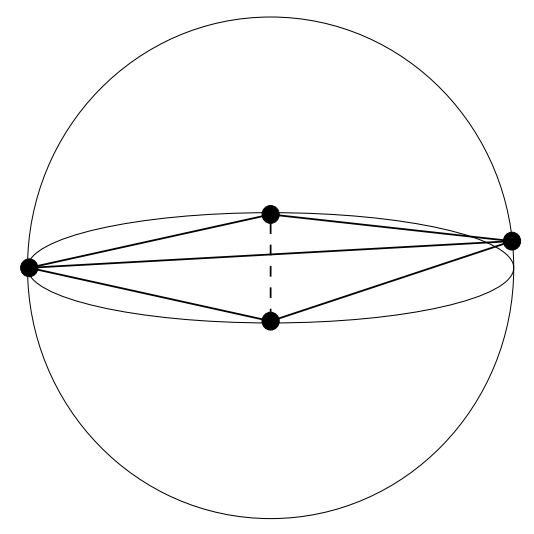

FIG. 1. Sliver and circumsphere.

inequality which will imply convergence of the control volume method in $L^{2}(\Omega)$.

These results evolved from our work on mesh generation algorithms $[6,7]$ and an attempt to resolve the problems slivers present in approximation theory. The mesh generation algorithms we developed produce tetrahedra having bounded ratios of circumscribing radius to minimum edge length (radius-edge ratio), while traditional interpolation theory requires each tetrahedra to have the classical aspect ratio of diameter to inscribed sphere radius bounded. In two dimensions, these two ratios are equivalent in the sense that each ratio is bounded above and below by a multiple of the other. In three dimensions, slivers, which have a radius-edge ratio near unity and arbitrarily small volume, show that a bounded radius-edge aspect ratio is a weaker condition than the classical aspect ratio in higher dimensions; moreover, they are the only tetrahedra for which the ratios are incomparable. While a bound on the classical aspect ratio is sufficient for optimal interpolation, it is not necessary. Babuška and Aziz [1] showed that right triangles in two dimensions, and their analogues in three dimensions, will exhibit optimal interpolation even if they have arbitrarily small volume. In two dimensions this leads to the very simple geometric requirement that "the maximum angle within any triangle be bounded away from $\pi$ "; however, no simple geometric restriction is known for tetrahedra. Upon discovering that the covolume scheme converges in the presence of slivers, it is natural to ask if they too exhibit optimal interpolation properties. Folklore indicates that they do not, and this is substantiated by the numerical example in section 3 .

In order to put the geometric assumptions into context, section 2 reviews the essential features of the mesh generation algorithm developed in [7]. As indicated above, this algorithm does guarantee a bound on the radius-edge aspect ratio. The proof of convergence for Poisson's equation under the bounded radius-edge ratio assumption is presented in section 3 along with an example which highlights the differences between the covolume and finite element schemes. In section 4 a discrete Poincaré inequality is established which provides one method of passing from mesh-dependent norms to the $L^{2}(\Omega)$ norm.

2. Sphere packings and mesh generation. Mesh generation has a long history; however, rigorous analysis of the algorithms and the meshes they produce is a recent development. Bern, Eppstein, and Gilbert [2] were the first to establish opti- 
mality of the quad-tree algorithm for the generation of two-dimensional meshes. By optimal we mean that bounds on the aspect ratio of each triangle can be established apriori and that the number of triangles produced is within a constant of the minimum possible. Unfortunately the constant for the quad-tree algorithm appears to be rather large, so that this, and its three-dimensional counterpart [8], is not a particularly practical approach. An optimal two-dimensional algorithm that appears to work very well in practice was given by Ruppert [10]. This algorithm extended an idea of Chew [3], and uses the Voronoi/Delaunay constructions. Ruppert's algorithm doesn't extend immediately to three dimensions since it may produce "slivers," that is, very flat tetrahedra whose edge lengths are comparable with the circumsphere radius (see Figure 1). These flat tetrahedra are difficult to eliminate and plague three-dimensional Delaunay-based mesh generation algorithms. In section 3 we establish the rather unexpected result that these slivers do not degrade control volume approximations of Poisson's equation, and in this situation extensions of Ruppert's algorithm can be used to produce meshes that are "provably good for control volume approximations." The remainder of this section introduces the basic geometric properties needed for the analysis in sections 3 and 4 .

Below we consider meshes generated as the Delaunay diagrams of a set of points $X=\left\{x_{i}\right\} \subset \bar{\Omega} \subset \mathbb{R}^{d}$ (we are particularly interested in three dimensions where $d=3$ ). Recall that the Voronoi diagram is the collection of polytopes $\left\{V_{i}\right\}$ where $V_{i}$ is the subset of points in $\Omega$ closer to $x_{i}$ than to any other point in the set $X$. The Delaunay triangulation is then constructed by joining points in $X$ that share a common Voronoi face. This construction is well known in computational geometry [4], and there are efficient algorithms for implementing it in any number of dimensions.

The meshes we consider below assume that the triangulation satisfies the following weakened aspect ratio condition.

DEFINITION 2.1 (bounded radius-edge ratio). The radius-edge ratio of a triangulation in three dimensions is the maximum ratio of circumscribing sphere radius to smallest edge length of any tetrahedron. (Recall that the circumscribing sphere is the sphere passing through the four vertices of the tetrahedron.)

We briefly sketch one technique for generating a vertex set whose Delaunay triangulations have bounded radius-edge ratios. We assume that we're given a function $\rho: \Omega \rightarrow \mathbb{R}^{+}$which locally specifies the desired density of mesh points in $\Omega$.

Definition 2.2. A collection of points $X=\left\{x_{1}, x_{2}, \ldots, x_{N}\right\} \subset \Omega$ is a $\rho$-packing if $x_{i}, x_{j} \in X$ implies $\left[\rho\left(x_{i}\right)+\rho\left(x_{j}\right)\right] / 2 \leq\left|x_{i}-x_{j}\right|$ where $|$.$| denotes Euclidean distance.$

A simple algorithm for the construction of a maximal $\rho$-packing is to initialize $X$ to be the empty set and to randomly select a point $x \in \Omega$ and add it to $X$ if the sphere of radius $\rho(x)$ centered at $x$ doesn't meet the analogous spheres of any point already in $X$. Upon termination, $X$ will be a maximal $\rho$-packing. The following lemma concerning the Voronoi/Delaunay diagram for $X$ is elementary.

Lemma 2.3. Let $\rho: \Omega \rightarrow \mathbb{R}^{+}$be Lipschitz ${ }^{2}$ with $|\rho|_{\text {Lip }}<2$ and let $X$ be a maximal $\rho$-packing. If $v$ is the center of a Delaunay ball (i.e., the circumscribing sphere of a tetrahedron) of radius $r$ in the Voronoi/Delaunay triangulation of $X$, then $\rho(v) \geq\left(1-|\rho|_{\text {Lip }} / 2\right) r$.

This lemma and Corollary 2.4 are proved in [6, 7], and provide a wealth of geometric information about such meshes.

Corollary 2.4. Let $\alpha=|\rho|_{\text {Lip }}<2 / 3$. Then

${ }^{2}$ Recall that $\rho$ is Lipschitz with constant $|\rho|_{\text {Lip }} \leq \alpha$ if $|\rho(x)-\rho(y)| \leq \alpha|x-y|$ for all $x$ and $y$. 
- If $v$ and $r$ are as in Lemma 2.3 and $x \in B_{r}(v)$, the ball or radius $r$ centered at $v$, then $\rho(x) \geq(1-3 \alpha / 2) r$.

- The radius-edge ratio of the Delaunay triangulation of $X$ is bounded by $1 /(1-$ $3 \alpha / 2)$.

- If $V$ is a Voronoi polytope centered at $x \in X$, the ratio of circumscribing radius of $V$ to inscribed radius of $V$ (with center $x$ ) is bounded by $(2+\alpha) /(1-$ $3 \alpha / 2)$.

- If $x, y \in X$ and $p \in \overline{x y}$ (an edge in the Delaunay diagram), then

$$
\rho(p) \geq \frac{1-3 \alpha / 2}{2+\alpha} \rho(x)
$$

- For any Voronoi region $V$

$$
\frac{\max _{V} \rho}{\min _{V} \rho} \leq \frac{(2+\alpha)(2+3 \alpha / 2)}{(1-3 \alpha / 2)^{2}}
$$

This corollary shows that the simple algorithm sketched above will produce tetrahedra with a bounded radius-edge ratio. Of all the tetrahedra that fail to have a bounded classical aspect ratio (needles, caps, etc.), the bounded radius-edge ratio eliminates all but the slivers. Currently there are not any Delaunay based mesh generation codes that can guarantee eliminating slivers.

The development of ideas sketched in this section began with a spacing function $\rho$ and led to a mesh with a bounded radius-edge ratio. It is also possible to do the converse. Theorem 4.4 in section 4 shows that a Delaunay mesh having bounded radius-edge ratio determines a Lipschitz spacing function $\rho$ satisfying the properties stated in the corollary.

\section{Covolume approximations.}

3.1. Notation. Let $\Omega \subset \mathbb{R}^{3}$ be a bounded open set, and consider the problem of finding $u: \Omega \rightarrow \mathbb{R}$ such that

$$
-\Delta u=f \quad \text { in } \Omega,\left.\quad u\right|_{\partial \Omega}=g .
$$

To approximate $u, \Omega$ is first triangulated, the triangulation being the Delaunay triangulation of a set of points $\left\{x_{i}\right\}$. We denote the length of the Delaunay edge joining vertex $x_{i}$ to $x_{j}$ by $h_{i j}$, and denote by $V_{i}$ the Voronoi region associated with node $x_{i}$. To minimize technical details we assume that the mesh accommodates the boundary in a fashion guaranteeing that the Voronoi $V_{i}$ corresponding to an interior vertex $x_{i}$ lies entirely within $\Omega$. Each edge $\overline{x_{i} x_{j}}$ of the Delaunay mesh can be associated with (i.e., is dual to) a face common to $V_{i}$ and $V_{j}$ which we denote by $A_{i j}$.

Following MacNeal [5], discrete approximations of $u$ are constructed by integrating the equation for $u$ over each Voronoi corresponding to an interior vertex:

$$
\begin{aligned}
\int_{V_{i}} f & =\int_{V_{i}}-\Delta u=\int_{\partial V_{i}}-\frac{\partial u}{\partial n} \\
& =\sum_{j \in \mathcal{N}_{i}} \int_{A_{i j}}-\frac{\partial u}{\partial n} \simeq \sum_{j \in \mathcal{N}_{i}}\left|A_{i j}\right| \frac{u_{i}-u_{j}}{h_{i j}} .
\end{aligned}
$$

In the above, $\mathcal{N}_{i}$ is the index set of the nodes connected to $x_{i}$ by an edge, $u_{i}$ is an approximation of $u\left(x_{i}\right)$, and $\left|A_{i j}\right|$ denotes the surface area of the Voronoi face $A_{i j}$. 
Letting $f_{i}$ denote the average value of $f$ over $V_{i}$ this scheme becomes

$$
\sum_{j \in \mathcal{N}_{i}}\left|A_{i j}\right| \frac{u_{i}-u_{j}}{h_{i j}}=\left|V_{i}\right| f_{i} \quad \forall x_{i} \text { interior, }
$$

and $u_{i}=g\left(x_{i}\right)$ for the boundary vertices.

3.2. Orthogonality and convergence. Nicolaides [9] introduced the following elegant analysis of the above scheme. First, note that the exact solution $u$ of the equation satisfies

$$
\begin{aligned}
\int_{V_{i}} f=\int_{V_{i}}-\Delta u & =\int_{\partial V_{i}} \frac{\partial u}{\partial n}=\sum_{j \in \mathcal{N}_{i}} \int_{A_{i j}}-\frac{\partial u}{\partial n} \\
\left|V_{i}\right| f_{i} & =\sum_{j \in \mathcal{N}_{i}}\left|A_{i j}\right| U_{i j}^{(2)}
\end{aligned}
$$

where $U_{i j}^{(2)}$ is the average value of the flux $-\partial u / \partial n$ on the face $A_{i j}$. Upon subtracting this from the equation satisfied by the discrete solution we obtain

$$
0=\sum_{j \in \mathcal{N}_{i}}\left|A_{i j}\right|\left(U_{i j}-U_{i j}^{(2)}\right)
$$

where $U_{i j}=\left(u_{i}-u_{j}\right) / h_{i j}$ is the discrete flux.

The second step is to consider an arbitrary set of nodal values $\left\{v_{i}\right\}$ that vanish on the boundary. Multiplying the above equation by $v_{i}$ and summing over all of the interior nodes gives

$$
\begin{aligned}
0 & =\sum_{i} \sum_{j \in \mathcal{N}_{i}}\left|A_{i j}\right|\left(U_{i j}-U_{i j}^{(2)}\right) v_{i} \\
& =\sum_{\overline{x_{i} x_{j}}}\left|A_{i j}\right|\left(U_{i j}-U_{i j}^{(2)}\right)\left(v_{i}-v_{j}\right) \\
& =\sum_{\frac{x_{i} x_{j}}{}}\left|A_{i j}\right| h_{i j}\left(U_{i j}-U_{i j}^{(2)}\right) V_{i j}
\end{aligned}
$$

where $\sum_{\overline{x_{i} x_{j}}}$ indicates summation over the nonboundary edges, and $V_{i j}=\left(v_{i}-\right.$ $\left.v_{j}\right) / h_{i j}$. Defining the inner product $[., .]_{W}$ on quantities defined upon the nonboundary edges by

$$
[U, V]_{W}=\sum_{\bar{x}_{i} x_{j}}\left|A_{i j}\right| h_{i j} U_{i j} V_{i j}
$$

we may write $\left[U-U^{(2)}, V\right]_{W}=0$ where it is understood that $U$ is the discrete flux given by the covolume scheme and the discrete flux $V$ must be the discrete gradient of a piecewise linear function $v$ vanishing on the boundary, i.e., $V_{i j}=\left(v_{i}-v_{j}\right) / h_{i j}$.

An error estimate can now follow by recognizing that the above orthogonality relationship states that the covolume approximation is the best projection of the flux $U^{(2)}$ onto the space of fluxes generated by discrete functions vanishing on the boundary. In particular, if we let $U_{i j}^{(1)}=\left(u\left(x_{i}\right)-u\left(x_{j}\right)\right) / h_{i j}$ be the flux determined by the interpolant of the exact solution, we obtain

$$
\left\|U-U^{(1)}\right\|_{W} \leq\left\|U^{(2)}-U^{(1)}\right\|_{W} .
$$


The following theorem characterizes the size of the approximation error on the righthand side of this expression.

THEOREM 3.1. Let $^{3} u \in H^{2}(\Omega)$ and $U_{i j}^{(1)}=\left(u\left(x_{i}\right)-u\left(x_{j}\right)\right) / h_{i j}$ be the approximation of the flux $U_{i j}^{(2)}=\left(-1 / A_{i j}\right) \int_{A_{i j}} \partial u / \partial n$. Then

$$
\left\|U^{(2)}-U^{(1)}\right\|_{W} \leq \frac{1}{2} \frac{\max }{\bar{x}_{i} x_{j}}\left[1+4\left(r_{i j} / h_{i j}\right)^{2} h_{i j}\right]\left\|D^{2} u\right\|_{L^{2}(\Omega)},
$$

where $r_{i j}$ is maximum radius of a control volume having $A_{i j}$ as a face. In particular, if the mesh has a bounded radius-edge ratio the error in the covolume scheme is given bounded by

$$
\left\|U-U^{(1)}\right\|_{W} \leq \frac{1}{2}\left(1+4 B^{2}\right)\left\|D^{2} u\right\|_{L^{2}(\Omega)} h,
$$

where $h=\max _{\overline{x_{i} x_{j}}} h_{i j}$.

Proof. Let us write

$$
\left\|U^{(2)}-U^{(1)}\right\|_{W}^{2}=\sum_{\bar{x}_{i} x_{j}}\left|A_{i j}\right| h_{i j} \mu_{i j}(u)^{2}
$$

where

$$
\mu_{i j}(u)=\frac{-1}{A_{i j}} \int_{A_{i j}} \frac{\partial u}{\partial n}-\frac{u\left(x_{i}\right)-u\left(x_{j}\right)}{h_{i j}} .
$$

We explicitly compute the term $\mu_{i j}(u)$ for a generic edge $h_{i j}$. It is convenient to put the origin at the middle of $h_{i j}$ and let $h_{i j}$ lie in the $z$-axis so that $A_{i j}$ lies in the plane $z=0$, and for definiteness let $h_{i j}$ point from $x_{i}$ to $x_{j}$ up the $z$-axis (see Figure 2). Next, let $K_{i j}^{+}$be the cone with vertex $x_{j}$ and base $A_{i j}$ and $K_{i j}^{-}$be the cone with vertex $x_{i}$ and base $A_{i j}$, and note that cones corresponding to different Voronoi faces $A_{i j}$ are disjoint, and the union of all such cones is the union of the Voronoi regions $V_{i}$ corresponding to internal vertices $x_{i}$ (Figure 2).

For the calculations below we drop the subscript $i j$, so that $h=h_{i j}, A=A_{i j}$, etc. The cone $K^{+}$may be parameterized by

$$
x=(1-2 \eta / h) \xi_{1}, \quad y=(1-2 \eta / h) \xi_{2}, \quad z=\eta,
$$

$\left(\xi_{1}, \xi_{2}\right) \in A, 0 \leq \eta \leq h / 2$. Note that

$$
\int_{K^{+}} u=\int_{A} \int_{0}^{h / 2} u\left(\xi_{1}, \xi_{2}, \eta\right)(1-2 \eta / h)^{2} d \eta d \xi
$$

where $d \xi=d \xi_{1} d \xi_{2}$. Also

$$
\begin{gathered}
\frac{\partial u}{\partial \eta}=-2 \frac{\xi_{1}}{h} \frac{\partial u}{\partial x}-2 \frac{\xi_{2}}{h} \frac{\partial u}{\partial y}+\frac{\partial u}{\partial z} \\
\frac{\partial^{2} u}{\partial \eta^{2}}=\left(-2 \xi_{1} / h,-2 \xi_{2} / h, 1\right)\left[D_{x}^{2} u\right]\left(-2 \xi_{1} / h,-2 \xi_{2} / h, 1\right)^{T}, \\
\left|\frac{\partial^{2} u}{\partial \eta^{2}}\right| \leq\left[1+4\left(\xi_{1}^{2}+\xi_{2}^{2}\right) / h^{2}\right]\left|D^{2} u\right| .
\end{gathered}
$$

${ }^{3} H^{2}(\Omega)$ is the set of functions in $L^{2}(\Omega)$ having all second derivatives in $L^{2}(\Omega)$. 


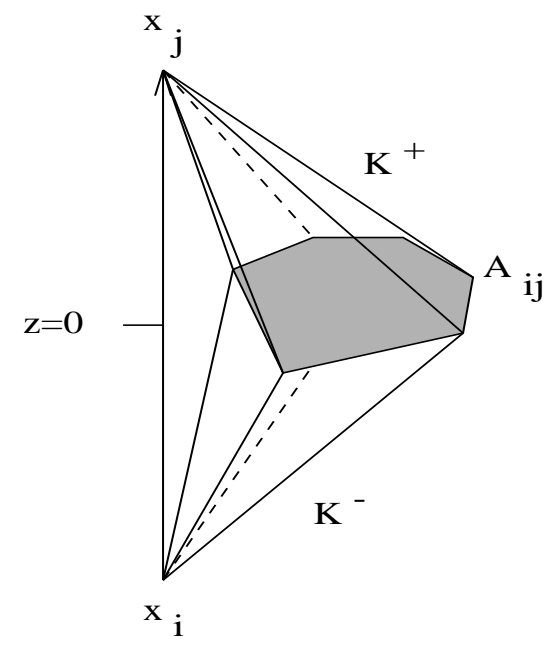

FIG. 2. Cones constructed in Theorem 3.1.

We now compute

$$
\begin{aligned}
\int_{K^{+}} \frac{1}{1-2(\eta / h)} \frac{\partial^{2} u}{\partial \eta^{2}} & =\int_{A} \int_{0}^{h / 2} \frac{\partial^{2} u}{\partial \eta^{2}}(1-2 \eta / h) d \eta d \xi \\
& =\int_{A}\left\{\left.\frac{\partial u}{\partial \eta}(1-2 \eta / h)\right|_{0} ^{h / 2}+\frac{2}{h} \int_{0}^{h / 2} \frac{\partial u}{\partial \eta} d \eta\right\} d \xi \\
& =\int_{A}\left\{-\frac{\partial u}{\partial \eta}\left(\xi_{1}, \xi_{2}, 0^{+}\right)+\frac{2}{h}\left(u\left(x_{j}\right)-u\left(\xi_{1}, \xi_{2}, 0^{+}\right)\right)\right\} d \xi \\
& =\int_{A}\left\{-\frac{\partial u}{\partial \eta}\left(\xi_{1}, \xi_{2}, 0^{+}\right)-2 u\left(\xi_{1}, \xi_{2}, 0^{+}\right) / h\right\} d \xi+2|A| u\left(x_{j}\right) / h .
\end{aligned}
$$

Repeat this computation for the bottom cone using the parameterization

$$
x=(1+2 \eta / h) \xi_{1}, \quad y=(1+2 \eta / h) \xi_{2}, \quad z=\eta,
$$

$\left(\xi_{1}, \xi_{2}\right) \in A,-h / 2 \leq \eta \leq 0$, to obtain

$$
\int_{K^{-}} \frac{1}{1+2(\eta / h)} \frac{\partial^{2} u}{\partial \eta^{2}}=\int_{A}\left\{\frac{\partial u}{\partial \eta}\left(\xi_{1}, \xi_{2}, 0^{-}\right)-2 u\left(\xi_{1}, \xi_{2}, 0^{-}\right) / h\right\} d \xi+2|A| u\left(x_{i}\right) / h .
$$

Continuity of $u$ guarantees that $u\left(., 0^{+}\right)=u\left(., 0^{-}\right)$so that

$$
\begin{aligned}
& \int_{K^{+}} \frac{1}{1+2(\eta / h)} \frac{\partial^{2} u}{\partial \eta^{2}}-\int_{K^{-}} \frac{1}{1-2(\eta / h)} \frac{\partial^{2} u}{\partial \eta^{2}} \\
& =-\int_{A}\left\{\frac{\partial u}{\partial \eta}\left(\xi_{1}, \xi_{2}, 0^{-}\right)+\frac{\partial u}{\partial \eta}\left(\xi_{1}, \xi_{2}, 0^{+}\right)\right\} d \xi-2|A| \frac{u\left(x_{i}\right)-u\left(x_{j}\right)}{h} .
\end{aligned}
$$

Note that $\partial u / \partial \eta=2\left(\xi_{1} / h\right) \partial u / \partial x+2\left(\xi_{2} / h\right) \partial u / \partial y+\partial u / \partial z$ in $K^{-}$so that

$$
\begin{aligned}
\int_{K^{+}} \frac{1}{1+2 \eta / h} \frac{\partial^{2} u}{\partial \eta^{2}}-\int_{K^{-}} \frac{1}{1-2 \eta / h} \frac{\partial^{2} u}{\partial \eta^{2}} & =2 \int_{A}-\left.\frac{\partial u}{\partial z}\right|_{z=0}-2|A| \frac{u\left(x_{i}\right)-u\left(x_{j}\right)}{h} \\
& =2|A| \mu(u) .
\end{aligned}
$$


It remains to bound the terms involving the second derivatives.

$$
\begin{aligned}
\left|\int_{K^{+}} \frac{1}{1-2(\eta / h)} \frac{\partial^{2} u}{\partial \eta^{2}}\right| & \leq\left|\int_{K^{+}} \frac{1+4\left(\xi_{1}^{2}+\xi_{2}^{2}\right) / h^{2}}{1-2(\eta / h)}\right| D^{2} u|| \\
& \leq\left\|\frac{1+4\left(\xi_{1}^{2}+\xi_{2}^{2}\right) / h^{2}}{1-2(\eta / h)}\right\|_{L^{2}\left(K^{+}\right)}\left\|D^{2} u\right\|_{L^{2}\left(K^{+}\right)} \\
& \leq(1 / 2) \sqrt{|A| h}\left[1+4(r / h)^{2}\right]\left\|D^{2} u\right\|_{L^{2}\left(K^{+}\right)},
\end{aligned}
$$

where $r^{2}=\max _{\left(\xi_{1}, \xi_{2}\right) \in A}\left(\xi_{1}^{2}+\xi_{2}^{2}\right)$. A similar computation for the lower cone gives

$$
|\mu(u)| \leq \frac{1}{2} \sqrt{\frac{h}{|A|}}\left[1+4(r / h)^{2}\right]\left\|D^{2} u\right\|_{L^{2}\left(K^{+} \cup K^{-}\right)} .
$$

Putting it all together gives

$$
\begin{aligned}
\left\|U-U^{(1)}\right\|_{W} & \leq\left\|U^{(2)}-U^{(1)}\right\|_{W}=\left(\sum_{\overline{x_{i} x_{j}}}\left|A_{i j}\right| h_{i j} \mu_{i j}(u)^{2}\right)^{1 / 2} \\
& \leq(1 / 2) \max _{\bar{x}_{i} x_{j}}\left[\left(1+4\left(r_{i j} / h_{i j}\right)^{2}\right) h_{i j}\right]\left(\sum_{\bar{x}_{i} x_{j}}\left\|D^{2} u\right\|_{L^{2}\left(K_{i j}^{+} \cup K_{i j}^{-}\right)}^{2}\right)^{1 / 2} \\
& \leq(1 / 2) \max _{\bar{x}_{i} x_{j}}\left[\left(1+4\left(r_{i j} / h_{i j}\right)^{2}\right) h_{i j}\right]\left\|D^{2} u\right\|_{L^{2}(\Omega)}
\end{aligned}
$$

3.3. Numerical example. We illustrate the implications of our theorem with a numerical example. Meshes are constructed by dividing the unit cube into a uniform rectangular mesh and then randomly perturbing the points by an amount $\epsilon h$. The Delaunay triangulation of such points will have multitudes of slivers on all of the vertical and horizontal planes where the points are almost cocircular. While the radius-edge ratio of these slivers will be near unity, their classical aspect ratio of diameter to inscribed sphere radius $(d / r)$ will be very large when $\epsilon$ is small. Figure 3 tabulates the errors for the solution

$$
u(x, y, z)=e^{\pi x} \cos (\pi y / \sqrt{2}) \sin (\pi z / \sqrt{2})
$$

of $-\Delta u=0$ computed using the finite element and covolume algorithms. Since the finite element solution is the best approximation of the solution in the $H_{0}^{1}(\Omega)$ norm, this is tabulated along with the norm $\|\cdot\|_{W}$ for the finite element solution.

It is clear that for fixed $h$ the finite element errors for each norm increase as $\epsilon \rightarrow 0$ and the the covolume error is independent of $\epsilon$. Divergence in the $H_{0}^{1}(\Omega)$ norm illustrates that even when the exact solution is interpolated at the nodal values, the presence of slivers causes large errors in the gradient. In order for the finite element method to achieve the smallest $H_{0}^{1}(\Omega)$ error possible it spreads this error among all tetrahedra, causing a failure of convergence in $\|\cdot\|_{W}$.

For $\epsilon$ fixed, the first-order rate of convergence for the finite element solution in the $H_{0}^{1}(\Omega)$ norm is readily observable (the error decreases in proportion with $h$ ). The covolume scheme achieves a higher rate of convergence (almost two) for this example since the mesh is almost uniform. On a uniform mesh the covolume scheme is, in fact, second-order accurate (this can readily be observed in the proof of Theorem 3.1). 


\begin{tabular}{c|lr|cccc} 
& & & \multicolumn{2}{|c}{ FEM } & CoVolume \\
$\mathrm{h}$ & $\epsilon$ & $d / r$ & $\left|u-u_{h}\right|_{H^{1}}$ & $\left\|I_{h} u-u_{h}\right\|_{W}$ & $\left\|I_{h} u-u_{h}\right\|_{W}$ \\
\hline $1 / 4$ & 0.01 & 1126 & 30.22479 & 7.696399 & 0.2425438 \\
& 0.001 & 11125 & 92.97309 & 8.692323 & 0.2411516 \\
& 0.0001 & 111296 & 291.6406 & 8.917005 & 0.2410718 \\
$1 / 8$ & 0.01 & 26230 & 12.98964 & 4.344101 & 0.07616430 \\
& 0.001 & 166051 & 35.13775 & 6.865007 & 0.07531520 \\
& 0.0001 & 1621745 & 108.1435 & 7.525118 & 0.07538803 \\
$1 / 16$ & 0.01 & 14194 & 6.023042 & 2.262189 & 0.02155074 \\
& 0.001 & 105551 & 15.48025 & 4.457009 & 0.02005826 \\
& 0.0001 & 1030956 & 45.91799 & 6.140328 & 0.02007369
\end{tabular}

FIG. 3. Errors for control volume and finite element methods.

4. Poincaré inequality. The Poincaré inequality states that for each bounded domain $\Omega \subset \mathbb{R}^{d}$ there is a constant $C>0$ such that $\|u\|_{L^{2}(\Omega)} \leq C\|\nabla u\|_{L^{2}(\Omega)}$ for all sufficiently smooth functions $u: \Omega \rightarrow \mathbb{R}$ vanishing on the boundary $\partial \Omega$. MacNeal [5] has shown that in two dimensions the $W$ norm (defined in section 3.2) is equal to the $H^{1}(\Omega)$ norm, in the sense that the piecewise linear extension, $\tilde{u}$, of $u$ defined on the vertices of a triangulation satisfies $\|u\|_{W}^{2}=\int_{\Omega}|\nabla \tilde{u}|^{2}$. The Poincaré inequality, $\int_{\Omega} u^{2} \leq C \int_{\Omega}|\nabla u|^{2}$ then implies that convergence of a discrete solution in the $W$ norm implies convergence in $L^{2}(\Omega)$. In three dimensions the $W$ norm is no longer equivalent to the $H^{1}(\Omega)$ norm, so convergence in $L^{2}(\Omega)$ doesn't follow directly from the Poincaré inequality. Below we establish a discrete Poincaré inequality for the $W$ norm which establishes convergence in $L^{2}(\Omega)$ of the functions that are piecewise constant on the Voronoi cells and take the nodal values of $u$.

Definition 4.1. Given a real valued function $u$ on the mesh vertices, the piecewise constant extension $\hat{u}: \Omega \rightarrow \mathbb{R}$ is defined to be the function equal to $u_{i}$ over the Voronoi cell $V_{i}$.

The discrete $L^{2}(\Omega)$ norm of $u$ is then interpreted to mean $\|\hat{u}\|_{L^{2}(\Omega)}^{2}=\sum_{i} \int_{V_{i}} u_{i}^{2}=$ $\sum_{i}\left|V_{i}\right| u_{i}^{2}$.

TheOREm 4.2 (discrete Poincaré inequality). Let $u$ be a function defined on the vertices of a triangulation of the bounded domain $\Omega \subset \mathbb{R}^{d}$ that vanishes on the boundary vertices. Then there exists $C>0$ depending only upon $\Omega$ and the radius-edge ratio of the mesh such that $\|u\|_{L^{2}(\Omega)} \leq C\|u\|_{W}$.

The theorem statement can be rewritten explicitly as

$$
\sum_{i}\left|V_{i}\right| u_{i}^{2} \leq C \sum_{\overline{x_{i} x_{j}}} \frac{\left|A_{i j}\right|}{h_{i j}}\left(u_{i}-u_{j}\right)^{2}
$$

for all mesh functions vanishing on the boundary. We adopt here the notation of the previous section. In particular, $\bar{x}_{i} x_{j}$ indexes the edges in the mesh whose interiors lie in $\Omega, h_{i j}$ is the edge length, and $A_{i j}$ is the area of the dual face.

Note that the left-hand side of this equation is the $L^{2}(\Omega)$ norm of the piecewise constant function on the Voronoi's, and in two dimensions the right-hand side is $\left\|\nabla u_{h}\right\|_{L^{2}(\Omega)}^{2}$ where $u_{h}$ is the piecewise linear function taking on the corresponding nodal values. Thus in two dimensions, with regular meshes, this inequality will follow from approximation theory. We show that this inequality continues to hold for meshes in two or three dimensions under the stated assumptions; in particular, even if slivers are present. 
4.1. Graphs and meshes. The meshes we consider are constructed from Delaunay diagrams, which are determined by a set of vertices $X=\left\{x_{i} \cdots x_{n}\right\}$, a set of tetrahedra whose 1-skeleton is the edge set $E$, and a set of Voronoi cells $V$. We define the following weighted graph associated with the mesh.

Definition 4.3. 1. Let $\mathcal{G}=\mathcal{G}(X, E, V)$ denote the graph constructed from the Delaunay diagram $\mathcal{D}=(X, E, V)$ as follows:

$X$ : The vertex set of the graph is identified with the vertex set of the Delaunay diagram.

E: Edges $(i, j)$ in the graph correspond to interior Delaunay edges and have weight $k_{i j}=\left|A_{i j}\right| / h_{i j}$. Recall $h_{i j}$ is the length of the Delaunay edge between two vertices, $A_{i j}$ is the Voronoi face shared by $V_{i}$ and $V_{j}$.

$V$ : We assign the Delaunay cell volume to each graph vertex.

2. The mass matrix $M$ is a diagonal matrix containing the (truncated) Voronoi volumes on the diagonal. (Recall that Voronoi regions on the boundary may be infinite, so we only consider the part in $\Omega$. We treat the areas used for computing the edge weights in a similar fashion. These terms are inconsequential since we only consider functions vanishing on the boundary.)

3. The Laplacian of the graph $\mathcal{G}$, denoted by $K$, is the matrix having off diagonal entries $K_{i j}=-\left|A_{i j}\right| / h_{i j}=-k_{i j}$ if $(i, j)$ is an edge in $\mathcal{G}$ and zero otherwise and the diagonals are given by $K_{i i}=\sum_{j \neq i} k_{i j}$.

The discrete Poincaré inequality then states that the eigenvalues (on the space of vectors having zero boundary components) of the generalized eigenvalue problem $K u=\lambda M u$ are bounded below by a constant $c>0$, or alternatively that there is a constant $c>0$ such that $K-c M \geq 0$ (positive semidefinite).

4.2. Geometric properties. The meshes we consider are Delaunay diagrams $\mathcal{D}=(X, E, V)$ where $X \subset \bar{\Omega} \subset \mathbb{R}^{d}$. We assume the mesh tetrahedra are of bounded radius-edge ratio.

Delaunay diagrams of bounded radius-edge ratio have several important geometric properties. Below we recall some of these properties which were established in [6]. Recall that we denote the edge joining two vertices $x_{i}$ and $x_{j}$ by $\bar{x}_{i} x_{j}$ and the length of such an edge by $h_{i j}$ and that the set of vertices connected to vertex $x_{i}$ by an edge is denoted by $\mathcal{N}_{i}$. Also, we adopt the notation that $C$ and $c$ are positive constants which may differ from occurrence to occurrence. In general, $c$ will be a lower bound and $C$ an upper bound, and these constants depend only upon the mesh through the Lipschitz constant of the spacing function, $\rho$, appearing in the following theorem.

TheOREM 4.4. Let $\mathcal{D}=(X, E, V)$ be a bounded radius-edge ratio Delaunay diagram. The following is true.

A1. There is a function $\rho: \Omega \rightarrow \mathbb{R}^{+}$with Lipschitz constant bounded by one and constants $c, C>0$ such that for each internal vertex $x_{i} \in X$

$$
c h_{i j} \leq \rho(x) \leq C h_{i j} \quad \forall j \in \mathcal{N}_{i}, x \in V_{i}
$$

and for all edges $\bar{x}_{i} x_{j}$

$$
c h_{i j} \leq \rho(x) \leq C h_{i j} \quad \forall x \in \bar{x}_{i}{ }_{j} .
$$

A2. There is a constant $\theta>0$ such that each region $V_{i} \cap \Omega$, where $V_{i} \in V$, contains a ball of radius $\theta \rho\left(x_{i}\right)$ centered at $x_{i}$ and is contained in a concentric ball of radius $\rho\left(x_{i}\right) / \theta$.

A3. There is a constant $C>0$ such that each vertex $x_{i} \in X$ has at most $C$ neighbors in the diagram, i.e., the associated graph has bounded degree. 
A4. There is a constant $C>0$ such that the area of the Voronoi face dual to the vertices $x_{i}, x_{j} \in X$ is bounded from above by $C h_{i j}^{d-1}$.

All of the constants depend upon the mesh solely through the the Lipschitz constant of the spacing function $\rho$.

The function $\rho$ is referred to as the spacing function of the mesh, as it describes the typical distance between vertices. This function plays an important role in the proofs below as it captures many of the mesh properties.

Our arguments below are graph theoretic and don't use the fact that we're dealing with a Voronoi/Delaunay triangulation; any decomposition of $\Omega$ into volumes $V_{i}$ and edges connecting them that satisfy the geometric properties listed in Theorem 4.4 would suffice. We therefore generalize the notion of the bounded radius-edge ratio Delaunay diagram as follows.

Definition 4.5 (Well-shaped diagram). A diagram $\mathcal{G}=(X, E, V)$ is a set of vertices $X$, and a set of cells $V$ such that vertex $x_{i} \in V_{i}$, and the edge set $E$ corresponds to the neighborhood structure of the cells. The diagram is well shaped if properties A1-A4 hold.

Notation. Since the edges of a well-shaped diagram don't necessarily correspond to line segments in $\mathbb{R}^{d}$ we will denote an edge connecting $x_{i}, x_{j} \in X$ by $(i, j)$ and use the notation $\bar{x}_{i} x_{j}$ to suggest a line segment in $\mathbb{R}^{d}$.

4.3. Comparison. Our proof of the discrete Poincaré inequality will parallel the proof of its continuous counterpart. The continuous proof considers a large cube $Q$ containing $\Omega$, and since all functions defined on $\Omega$ vanishing on the boundary can be extended by zero to all of $Q$, a Poincaré inequality on $Q$ will imply a Poincaré inequality on $\Omega$. We too will embed $\Omega$ into a large cube, and in this section we show that any two meshes on $\Omega$ and $Q$ satisfying the geometric properties of Theorem 4.4 with the same spacing function $\rho$ can be suitably compared. The next section will then show that for each spacing function there exists a canonical mesh on the cube for which the Poincaré inequality holds.

Definition 4.6 (Path embedding). 1. The graph $\mathcal{G}_{1}=\left(X^{(1)}, E^{(1)}, V^{(1)}\right)$ is path embedded in $\mathcal{G}_{2}=\left(X^{(2)}, E^{(2)}, V^{(2)}\right)$ if there exists a function $\phi$ from $X^{(1)}$ to $X^{(2)}$ and a mapping $p$ from the edges of $\mathcal{G}_{1}$ into paths in $\mathcal{G}_{2}$, such that for each edge $(m, n)$ in $\mathcal{G}_{1}, p(m, n)$ is a path in $\mathcal{G}_{2}$ from $\phi(m)$ to $\phi(n)$.

2. The embedding has bounded dilation if there is a constant $C_{k}>0$ such that for every edge $(m, n) \in E^{(1)}$,

$$
\sum_{(i, j) \in p(m, n)} 1 / k_{i j}^{(2)} \leq C_{k} / k_{m n}^{(1)}
$$

where $k_{m n}^{(1)}$ and $k_{i j}^{(2)}$ are the edge weights of $\mathcal{G}_{1}$ and $\mathcal{G}_{2}$ respectively (i.e., the conductivity of the path is comparable to the conductivity of the edge).

3. The embedding has bounded congestion if there is a constant $C_{p}>0$ such that for each edge $(i, j)$ in $\mathcal{G}_{2}$

$$
c_{i j}=\sum_{p(m, n) \ni(i, j)} 1 \leq C_{p}
$$

Lemma 4.7. Let $(\phi, p)$ be a path embedding of $\mathcal{G}_{1}$ into $\mathcal{G}_{2}$ with bounded dilation and congestion, and let $u$ be a real valued function on the vertices of $\mathcal{G}_{2}$ and $u \circ \phi$ be the induced function on the vertices of $\mathcal{G}_{1}$. Then there is a constant $C>0$ such that

$$
(u \circ \phi)^{T} K^{(1)}(u \circ \phi) \leq C u^{T} K^{(2)} u .
$$


Proof.

$$
\begin{aligned}
(u \circ \phi)^{T} K^{(1)}(u \circ \phi) & =\sum_{(m, n)} k_{m n}^{(1)}\left(u_{\phi(m)}-u_{\phi(n)}\right)^{2} \\
& =\sum_{(m, n)} k_{m n}^{(1)}\left(\sum_{(i, j) \in p(m, n)}\left(u_{i}-u_{j}\right)\right)^{2} \\
& \leq \sum_{(m, n)} k_{m n}^{(1)}\left(\sum_{(i, j) \in p(m, n)} 1 / k_{i j}^{(2)}\right) \sum_{(i, j) \in p(m, n)} k_{i j}^{(2)}\left(u_{i}-u_{j}\right)^{2} \\
& \leq C_{k} \sum_{(m, n)(i, j) \in p(m, n)} k_{i j}^{(2)}\left(u_{i}-u_{j}\right)^{2} \\
& \leq C_{k} C_{p} \sum_{(i, j)} k_{i j}^{(2)}\left(u_{i}-u_{j}\right)^{2} .
\end{aligned}
$$

We plan to embed a cube $Q$ into $\Omega$ and need a way of extending functions on $\Omega$ that vanish on the boundary to all of $Q$. A convenient way of doing this is to add a "super" node $x_{0}$ to the vertex set $X$ of a well-shaped diagram $\mathcal{G}=(X, E, V)$ of $\Omega$, and to augment the edge set $E$ by connecting every boundary vertex to the super node, and to assign large (say infinite) weights to these edges so that they never lower the conductivity of a path. Also, we may put multiple (say infinite) edges between a boundary node and the super node to guarantee that these edges never increase the congestion. It is convenient to associate the region $V_{0}=Q \backslash \Omega$ to the super node $x_{0}$.

Notation. Given a well-shaped diagram $\mathcal{G}=(X, E, V)$ of $\Omega$, the augmented diagram is the diagram formed by adjoining a super node to $X$ and the associated edges and volume to $E$ and $V$.

Property A4 allows the edge weights $A_{i j} / h_{i j}$ to be arbitrarily small. In particular, a sliver in a Delaunay diagram corresponds to a small weight. All the paths containing the "light" edge will have small conductivity. The following lemma states that this situation is not generic and that each edge can be replaced by a path of high conductivity, which can then be used to construct suitable path embeddings.

LEMmA 4.8. Let $\mathcal{G}=(X, E, V)$ be an augmented well-shaped diagram of $\Omega \subset \mathbb{R}^{d}$. Then there exists a path embedding $(\phi, p)$ of $\mathcal{G}$ into itself of bounded congestion such that the conductivity of each path in the image is bounded below by a multiple of $h_{i j}^{d-2}$, i.e., for each edge $(i, j) \in E$,

$$
\sum_{(m, n) \in p(i, j)} 1 / k_{m n} \leq C / h_{i j}^{d-2} .
$$

Proof. Let us consider a typical edge $\bar{x}_{i} x_{j}$ of length $h_{i j}$ and define $U$ to be the neighborhood of $\bar{x}_{i} x_{j}$ consisting of points whose distance from $\bar{x}_{i} x_{j}$ is no more than $\min (c / 2, \theta c) h_{i j}$ where $c$ and $\theta$ are the constants guaranteed by properties A1 and $A 2$ of Theorem 4.4. We first consider the case when $U$ lies entirely within $\Omega$. The Lipschitz hypotheses on $\rho$ guarantee that $c / 2 h_{i j} \leq \rho(x) \leq(C+c / 2) h_{i j}$ for all $x \in U$.

Step 1: The number of cells from $V$ that intersect $U$ is bounded by a constant $\kappa \geq 0$. 
We use a volume argument. If $x \in V_{k} \cap U$, property A1 shows that $c / C \leq$ $\rho\left(x_{k}\right) / \rho(x) \leq C / c$. Since $\rho(x)$ is bounded above and below by multiples of $h_{i j}$, property A2 implies that $V_{k}$ contains a ball having volume bounded below by $\hat{c} h_{i j}^{d}$ and the diameter of $V_{k}$ is bounded by $\hat{C} h_{i j}$. If $\tilde{U}$ is the set of points having distance no more than $\hat{C} h_{i j}$ from $\bar{x}_{i} x_{j}$, then $\tilde{U}$ contains all of the cells intersecting $U$ and has volume bounded by a multiple of $h_{i j}^{d}$. It follows that the number of cells meeting $U$ is bounded by $|\tilde{U}| / \hat{c} h_{i j}^{d}$.

Step 2: There exists a line segment from $V_{i}$ to $V_{j}$ in $U$, parallel to $\bar{x}_{i} x_{j}$, that meets Voronoi faces having areas bounded below by a multiple of $h_{i j}^{d-1}$.

We use an area argument similar in spirit to the previous volume argument. Consider the collection of line segments in $U$ that start in $V_{i}$ and end in $V_{j}$ and are parallel to $\bar{x}_{i} x_{j}$. By construction, the cross-sectional area of this collection is bounded below by a multiple of $h_{i j}^{d-1}$, say $\hat{c} h_{i j}^{d-1}$. Letting $\delta$ be the maximal degree of any vertex guaranteed by property $\mathrm{A} 3$, the total number of faces that meet $U$ is bounded by $\kappa \delta$. It follows that some path must encounter only faces having areas bounded below by $(\hat{c} / \kappa \delta) h_{i j}^{d-1}$ (otherwise every face would be small and their totality wouldn't exhaust the cross section).

We now observe that these conclusions don't change if a $\Omega$ is not convex and a portion of $U$ is exterior to $\Omega$. Since the volume of $U \cap \Omega$ is trivially smaller than that of $U$, the number of cells meeting $U$ only decreases in this case. Similar reasoning holds for the area argument. Note that all of the line segments do begin and end in $\Omega$ and that we consider all faces on the boundary to have large (infinite) area.

To establish the theorem we let $p(i, j)$ be the path in $E$ formed by connecting the Voronoi centers in the order encountered by a segment in $U$ which only intersects "large" Voronoi faces. This path has length at most $\kappa$, and since $\rho$ is bounded above and below by a constant times $h_{i j}$ so too are the lengths of all of edges in $p(i, j)$, which implies a bound on the conductivity of the path. Finally, note that an edge in $E$ will only be on a path $p(i, j)$ for vertices $x_{i}$ and $x_{j}$ that can be reached along no more than $\kappa$ edges. Since the degree of each vertex is bounded by the constant $\delta$, the congestion of any edge is less than $\delta^{\kappa}$.

The paths guaranteed by this technical lemma enable us to construct path embeddings between diagrams in the following theorem.

THEOREM 4.9. Let $\mathcal{G}_{1}=\left(X^{(1)}, E^{(1)}, V^{(1)}\right)$ be a well-shaped diagram for a cube $Q$ containing $\Omega$ and $\mathcal{G}_{2}=\left(X^{(2)}, E^{(2)}, V^{(2)}\right)$ be the augmentation of a well-shaped diagram of $\Omega$, each having the same spacing function $\rho$. Then there exists a path embedding $\mathcal{G}_{1}$ into $\mathcal{G}_{2}$ with congestion and dilation bounded by constants depending upon the Lipschitz constant of $\rho$.

Proof. Our proof proceeds by explicitly constructing a path embedding $(\phi, p)$ of $\mathcal{G}_{1}$ into $\mathcal{G}_{2}$, and then verifying that the congestion and dilation are appropriately bounded. The embedding is determined by the following construction (see Figure 4):

1. $\phi(m)=i$ where $V_{i}^{(2)} \in V^{(2)}$ is a cell intersecting the cell $V_{m}^{(1)} \in V^{(1)}$. (Note that $i$ is not uniquely specified as $V_{m}^{(1)}$ can intersect several cells of $V^{(2)}$.)

2. For each edge $(m, n) \in \mathcal{G}_{1}$ consider the three piecewise linear paths: $S_{1}=$ $\left(x_{\phi(m)}^{(2)}, \hat{x}_{0}, x_{m}^{(1)}\right), S_{2}=\left(x_{m}^{(1)}, x_{n}^{(1)}\right)$, and $S_{3}=\left(x_{n}^{(1)}, \hat{x}_{1}, x_{\phi(n)}^{(2)}\right)$ where $\hat{x}_{0} \in V_{m}^{(1)} \cap$ $V_{\phi(m)}^{(2)}$ and $\hat{x}_{1} \in V_{n}^{(1)} \cap V_{\phi(n)}^{(2)}$ (see Figure 4). Let $\ell_{1}=\phi(m), \ell_{2}, \ldots, \ell_{k-1}, \ell_{k}=$ $\phi(n)$ index the cells of $V^{(2)}$ encountered along the three segments $S_{1}, S_{2}, S_{3}$ in that order. Then $p(m, n)$ is the path in $\mathcal{G}_{2}$ formed by the union of the 


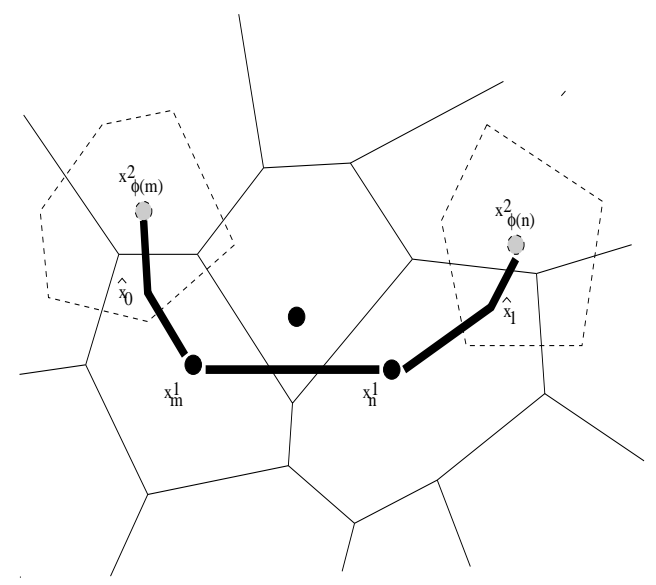

FIG. 4. Path constructed in Theorem 4.9.

heavily weighted paths from $x_{\ell_{i}}^{(2)}$ to $x_{\ell_{i+1}}^{(2)}$ constructed in Lemma 4.8.

We first note that this construction is well defined since the super node has the region $Q$ associated with it, so that every cell $V_{m} \in V^{(1)}$ meets some cell of $V^{(2)}$, and paths that leave $\Omega$ all go to the super node and can reenter anywhere from the super node.

Next, observe that every cell along the path $p(m, n)$ in $V^{(2)}$ intersects either $V_{m}^{(1)}$, $V_{n}^{(1)}$, or the edge joining them. Property A1 shows that $c h_{m n} \leq \rho(x) \leq C h_{m n}$ for every $x \in V_{m}^{(1)} \cup V_{n}^{(1)}$, which implies $\left.\left(c / C^{2}\right) h_{m n} \leq \rho(x) \leq C^{2} / c\right) h_{m n}$ for $x \in V_{\ell_{i}}^{(2)}$, $i=1,2, \ldots, k$.

An application of the volume argument used in the first step of Lemma 4.8 gives a bound on the length of the path $p(m, n)$. Briefly, the distance from any point in one of the cells $\left\{V_{\ell_{i}}^{(2)}\right\}_{i=1}^{k}$ to $S_{2}$ is bounded by a multiple of $h_{m n}$. This neighborhood of $S_{2}$ will have volume bounded by $\hat{C} h_{m n}^{d}$, and since each of the cells $V_{\ell_{i}}^{(2)}$ contains a ball having volume bounded below by a multiple of $h_{m n}^{d}$, say $\hat{c} h_{m n}$, it follows that there are no more than $\hat{C} / \hat{c}$ such cells. Since the heavily weighted paths constructed in Lemma 4.8 are all of bounded length, the length of $p(m, n)$ is bounded by at most a multiple of $\hat{C} / \hat{c}$.

This immediately leads to a bound on the congestion and dilation. Property A3 bounds the degree of any vertex by a constant $\delta$, so the congestion is bounded by the $L_{\max }^{\delta}$, where $L_{\max }$ is the maximal length of any path. Similarly, the conductivity on each edge is bounded below by a constant of the form $\hat{c} h_{m n}^{d-1}$ and putting $L_{\max }$ edges in the series doesn't decrease this by more than a factor of $1 / L_{\max }$.

Corollary 4.10. Let $\mathcal{G}_{1}=\left(X^{(1)}, E^{(1)}, V^{(1)}\right)$ and $\mathcal{G}_{2}=\left(X^{(1)}, E^{(2)}, V^{(2)}\right)$ be as in Theorem 4.9, and suppose that $Q$ is sufficiently large to guarantee that no boundary cell of $V^{(1)}$ meets a boundary cell of $V^{(2)}$. If $\mathcal{G}_{1}$ satisfies the Poincaré inequality, then so too does $\mathcal{G}_{2}$

Proof. Let $u$ be a real valued function on $X^{(2)}$ that vanishes on the boundary (and, in particular on the super node $x_{0}$ ). When constructing the path embedding $\mathcal{G}_{1}$ into $\mathcal{G}_{2}$ given by the theorem, select $\phi(m)$ in step 1 to correspond to the vertex 


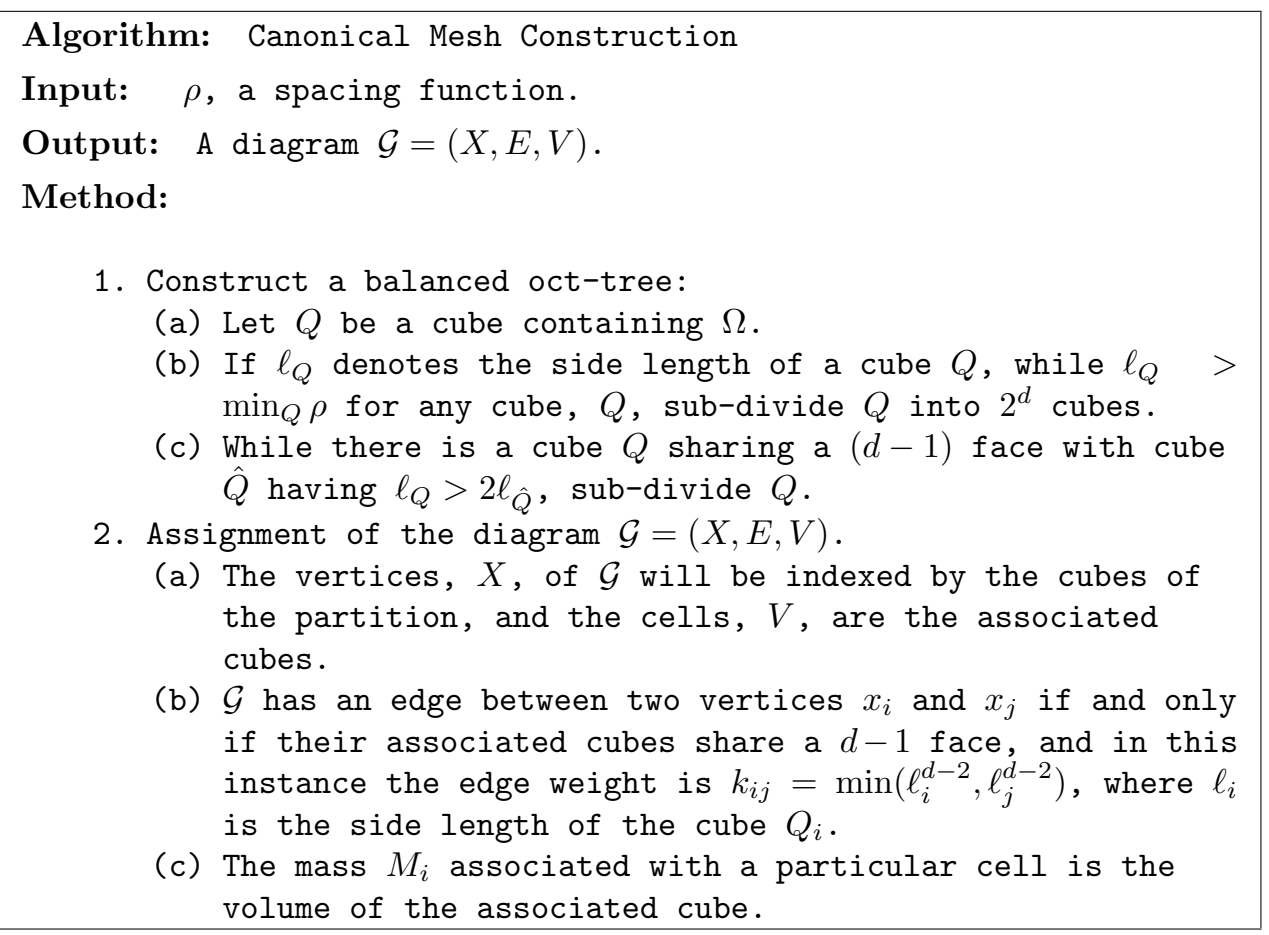

FIG. 5. Constructing a canonical mesh.

having maximal absolute value of $u$ of the available choices. It follows that

$$
\left|V_{i}^{(2)}\right| u_{i}^{2} \leq \sum_{m}\left|V_{m}^{(1)} \cap V_{i}^{(2)}\right| u_{\phi(m)}^{2},
$$

and summing over $i$ gives

$$
u^{T} M^{(2)} u=\sum_{i}\left|V_{i}^{(2)}\right| u_{i}^{2} \leq \sum_{m}\left|V_{m}^{(1)}\right| u_{\phi(m)}^{2}=(u \circ \phi)^{T} M^{(1)}(u \circ \phi) .
$$

Since all boundary vertices of $X^{(1)}$ are mapped by $\phi$ to the super node $x_{0}^{(2)}$, it follows that $u \circ \phi: X^{(1)} \rightarrow \mathbb{R}$ vanishes on the boundary vertices, and since $\mathcal{G}_{1}$ satisfies a Poincaré inequality we have

$$
u^{T} M^{(2)} u \leq(u \circ \phi)^{T} M^{(1)}(u \circ \phi) \leq C(u \circ \phi)^{T} K^{(1)}(u \circ \phi)
$$

and the result follows upon application of Lemma 4.7.

4.4. Construction of a graph satisfying a Poincaré inequality. In order to apply the results of the previous section to establish a Poincaré inequality for bounded well-shaped diagrams it is necessary to exhibit an instance of a canonical mesh satisfying a Poincaré inequality. We let $\rho: Q \rightarrow \mathbb{R}^{+}$be a specified Lipschitz spacing function, fixed during this discussion, and $Q$ a cube in $\mathbb{R}^{d}$.

Figure 5 exhibits the oct-tree construction for a canonical mesh, and the balancing step is illustrated in Figure 6. The oct-tree construction is well known, and the following lemma shows that our particular construction generates a well-shaped diagram with spacing function $\rho$. 

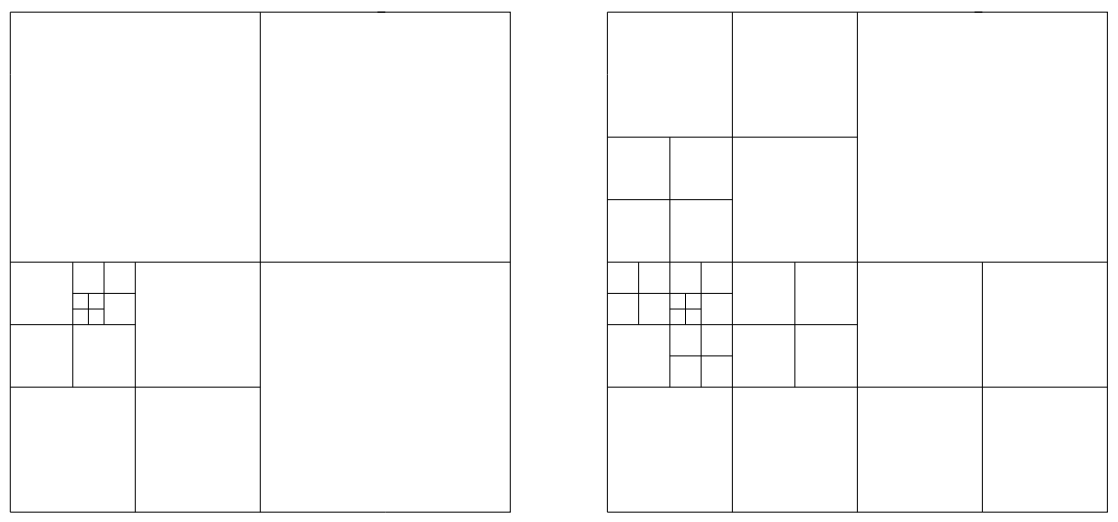

FIG. 6. Balancing a quad tree.

Lemma 4.11. The oct-tree diagram generated by the algorithm of Figure 5 is a well-shaped diagram with spacing function $\rho$.

Proof. Properties A3 and A4 follow immediately from the oct-tree construction. For properties A1 and A2, we only have to show the existence of constants $c, C>0$ such that each cube $Q$ in the balanced oct-tree generated by the algorithm of Figure 5 satisfies

$$
c \ell(Q) \leq \rho(x) \leq C \ell(Q), \quad x \in Q .
$$

Let $Q_{c}$ be a leaf cube at the end of step 1(b), and let $Q$ be its parent cube. Since $Q$ was divided there is some point $x \in Q$ such that $\rho(x) \leq \ell(Q)$, and since $Q_{c}$ was not divided, $\ell\left(Q_{c}\right)=\ell(Q) / 2<\rho(y)$ for all $y \in Q_{c}$. The distance between $x$ and $y$ is bounded by $|x-y| \leq \sqrt{d} \ell(Q)$, and since $\rho$ is $\alpha$-Lipschitz, $\rho(y) \leq \rho(x)+\alpha \sqrt{d} \ell(Q)$. In summary, by the end of step 1(b) of the algorithm,

$$
\ell\left(Q_{c}\right) \leq \rho(y) \leq 2(\alpha \sqrt{d}+1) \ell\left(Q_{c}\right), \quad y \in Q_{c} .
$$

We now show there are constants $c$ and $C$ such that after the balancing step 1(c) of the algorithm: $c \ell(Q) \leq \rho(y) \leq C \ell(Q)$ for $y \in Q$. Since subdividing a cube doesn't affect the lower bound on $\rho$, we set $c=1$. To establish the upper bound we use an induction argument. Our induction hypothesis is that prior to any balancing split, all cubes $Q$ satisfy $\rho(y) \leq C \ell(Q)$ for all $y \in Q$ ( $C$ is explicitly computed below).

We have shown that step $1(\mathrm{~b})$ produces cubes satisfying the inductive assumption provided $C \geq 2(\alpha \sqrt{d}+1)$, establishing the initial inductive step. Let $Q$ be a cube split during the balancing step, let $Q_{c}$ be one of its children, and let $\hat{Q}$ be a neighbor causing the split. It follows that $\ell(\hat{Q})<\ell(Q) / 2$, and if $x \in \hat{Q}$ the induction hypothesis guarantees that $\rho(x) \leq C \ell(\hat{Q})$. The distance between $x \in \hat{Q}$ and any $y \in Q$ is bounded by

$$
|x-y| \leq \sqrt{d}(\ell(\hat{Q})+\ell(Q)) \leq(3 \sqrt{d} / 2) \ell(Q) .
$$

Since $\rho$ is Lipschitz with constant $\alpha$,

$$
\begin{aligned}
\rho(y) & \leq \rho(x)+\alpha(3 \sqrt{d} / 2) \ell(Q) \\
& \leq(C / 2) \ell(Q)+\alpha(3 \sqrt{d} / 2) \ell(Q) \\
& \leq(C / 4+\alpha 3 \sqrt{d} / 4) \ell\left(Q_{c}\right) .
\end{aligned}
$$


Selecting $C$ such that $C / 4+\alpha 3 \sqrt{d} / 4 \leq C$ completes the inductive step.

The construction below mimics the proof of the Poincaré inequality for the continuous case which proceeds by integrating along a path from the boundary to each point in the domain. In the discrete case these paths must lie in the edge set of our graph and no one path can be used to excess (i.e., the congestion must be controlled; see Definition 4.6). The following is a generalization of path embedding of the previous section, where the edges we embed are from each internal vertex to the boundary vertices.

DeFINITION 4.12 (boundary embedding). Let $\mathcal{G}=(X, E, V)$ be a graph with edge weights denoted by $k_{i j}$. A boundary embedding is a collection of graphs $\left\{\mathcal{G}_{i}\right\}_{x_{i} \in X}$ each having the same vertex and edge structure of $\mathcal{G}$ and having edge weights $w_{m n}^{(i)} \geq 0$.

- The congestion of the boundary embedding is the maximum over all of the edges $(m, n) \in E$ of the sum $\sum_{i} w_{m n}^{(i)}$.

- For $x_{i} \in X$ the effective conductivity of $\mathcal{G}_{i}$ is the largest constant $c=c^{(i)}$ such that

$$
c u_{i}^{2} \leq \sum_{(m, n)} w_{m n}^{(i)} k_{m n}\left(u_{m}-u_{n}\right)^{2}
$$

for arbitrary nodal values $\left\{u_{m}\right\}$ vanishing on the boundary.

The role of these definitions becomes apparent from the following lemma.

LEMMA 4.13. Let $\mathcal{G}=(X, E, V)$ be a well-shaped diagram of $Q$ with mass matrix $M$ and Laplacian $K$. Let $\left\{\mathcal{G}_{i}\right\}$ be a boundary embedding having congestion bounded by $C$, and suppose that for each $\mathcal{G}_{i}$ the effective conductivity is bounded below by $M_{i}$. Then the Poincaré inequality $u^{T} M u \leq C u^{T} K u$ holds $\forall u: X \rightarrow \mathbb{R}$ vanishing on the boundary.

Proof. The bound on the conductivities guarantees that

$$
\begin{aligned}
u^{T} M u & =\sum_{i} M_{i} u_{i}^{2} \\
& \leq \sum_{i} \sum_{(m, n)} w_{m n}^{(i)} k_{m n}\left(u_{m}-u_{n}\right)^{2} \\
& \leq \sum_{(m, n)}\left(\sum_{i} w_{m n}^{(i)}\right) k_{m n}\left(u_{m}-u_{n}\right)^{2} \\
& \leq C u^{T} K u .
\end{aligned}
$$

We next construct the subgraphs $\mathcal{G}_{i}$ alluded to in Lemma 4.13. In the balanced oct-tree we define cube $Q_{i}$ to be above cube $Q_{j}$ if the intersection of their projections onto the plane $x_{d}=0$ have nonzero $(d-1)$ area. The horizontal faces of a cube are the two faces having $x_{d}$ constant. The key geometric fact used below is that if the intersection of the projections of two cubes has nonzero area, then the projection of the smaller cube must lie entirely within the projection of the larger cube (and the projections coincide if the cubes have the same side lengths).

Consider a vertex $x_{i}$ in $\mathcal{G}$ and define $\mathcal{G}_{i}$ to have the same vertex and edge sets as $\mathcal{G}$ and assign weights to the edges of $\mathcal{G}_{i}$ as follows: An edge $(m, n)$ has nonzero weight if and only if it is associated with a horizontal $d-1$ face and if $Q_{i}$ is above $Q_{m}$ and above $Q_{n}$. Without loss of generality we order the vertices so that $\ell\left(Q_{m}\right) \leq \ell\left(Q_{n}\right)$ 
and assign the edge weights by

$$
w_{m n}^{(i)}= \begin{cases}\ell\left(Q_{i}\right) & \text { if } \ell\left(Q_{i}\right)>\ell\left(Q_{m}\right), \\ \left(\frac{\ell\left(Q_{i}\right)}{\ell\left(Q_{m}\right)}\right)^{d-1} \ell\left(Q_{i}\right) & \text { otherwise. }\end{cases}
$$

THEOREM 4.14. The graph given by the above oct-tree construction satisfies a Poincaré inequality.

Proof. We show that the graphs $\left\{\mathcal{G}_{i}\right\}$ constructed above satisfy the hypotheses of Lemma 4.13.

Step 1: The congestion is bounded by $2 L$ where $L$ is the side length of $Q$.

A fixed (vertical) edge $\left(x_{m}, x_{n}\right)$ in $\mathcal{G}$, has a nonzero weight $w_{m n}^{(i)}$ if and only if the cube $Q_{i}$ lies above the cells $V_{m}$ and $V_{n}$. Without loss of generality, assume $\ell\left(Q_{m}\right) \leq \ell\left(Q_{n}\right)$. Let $I$ index all the cells $Q_{i}$ above $Q_{m}$ and $Q_{n}$. The congestion is then given by

$$
\begin{aligned}
c_{m n} & =\sum_{i \in I} w_{m n}^{(i)} \\
& =\sum_{i \in I, \ell\left(Q_{i}\right) \geq \ell\left(Q_{m}\right)} w_{m n}^{(i)}+\sum_{i \in I, \ell\left(Q_{i}\right)<\ell\left(Q_{m}\right)} w_{m n}^{(i)} \\
& =\sum_{i \in I, \ell\left(Q_{i}\right) \geq \ell\left(Q_{m}\right)} \ell\left(Q_{i}\right)+\sum_{i \in I, \ell\left(Q_{i}\right)<\ell\left(Q_{m}\right)} \ell\left(Q_{i}\right)^{d} / \ell\left(Q_{m}\right)^{d-1} .
\end{aligned}
$$

The first summand is bounded by $L$ since the larger cells must be stacked one on top of the other. The second summand is also less than $L$ since the sum gives the volume of the smaller cells above $Q_{m}$ divided by the cross-sectional area of $Q_{m}$.

Step 2: Let $L$ be the side length of $Q$; then the effective conductivity of $\mathcal{G}_{i}$ is bounded the below by $M_{i}=\left|V_{i}\right|$.

Let $\ell$ be the minimum side length of the cubes below $Q_{i}$ (which may be $\ell\left(Q_{i}\right)$ ), and think of the area of the projection of the cube $Q_{i}$ onto $x_{d}=0$ to be divided into squares of length $\ell$. The oct-tree construction guarantees that the projection of a cube $Q$ below $Q_{i}$ with $\ell(Q) \leq \ell\left(Q_{i}\right)$ will be the union of such squares, and if $Q$ is below $Q_{i}$ and $\ell(Q) \geq \ell\left(Q_{i}\right)$, then the intersection of the projections of $Q_{i}$ and $Q$ is equal to the projection of $Q_{i}$.

For each square $S$ in the projection, consider the path from the boundary to $Q_{i}$ formed by connecting the cubes below $Q_{i}$ that meet the cylinder $S \times \mathbb{R}$. For convenience label the vertices $m_{0}, m_{1}, \ldots, m_{I}$ so that $Q_{m_{0}}$ is a boundary cube and $Q_{i}=Q_{m_{I}}$. Then

$$
\begin{aligned}
|S| u_{i}^{2} & =|S|\left(\sum_{n=1}^{I}\left(u_{m_{n}}-u_{m_{n-1}}\right)\right)^{2} \\
& \leq \sum_{n=1}^{I} h_{m_{n} m_{n-1}} \sum_{n=1}^{I}\left(|S| / h_{m_{n} m_{n-1}}\right)\left(u_{m_{n}}-u_{m_{n-1}}\right)^{2} \\
& \leq L \sum_{n=1}^{I}\left(|S| / h_{m_{n} m_{n-1}}\right)\left(u_{m_{n}}-u_{m_{n-1}}\right)^{2}
\end{aligned}
$$

where $L$ is the side length of $Q$. Multiplying by $\ell\left(Q_{i}\right)$ and summing this inequality 
over all squares gives

$$
M_{i} u_{i}^{2} \leq L \sum_{S} \sum_{n=1}^{I_{s}}\left(\ell\left(Q_{i}\right)|S| / h_{m_{n} m_{n-1}}\right)\left(u_{m_{n}}-u_{m_{n-1}}\right)^{2},
$$

where $h_{m_{n} m_{n-1}}=\min \left(\ell\left(Q_{m_{n}}\right), \ell\left(Q_{m_{n-1}}\right)\right)$. If we define

$$
A_{m_{n} m_{n-1}}=\min \left(h_{m_{n} m_{n-1}}, \ell\left(Q_{i}\right)\right)^{d-1},
$$

interchanging the order of summation gives

$$
M_{i} u_{i}^{2} \leq L \sum\left(\ell\left(Q_{i}\right) A_{m_{n} m_{n-1}} / h_{m_{n} m_{n-1}}\right)\left(u_{m_{n}}-u_{m_{n-1}}\right)^{2},
$$

where the summation is over all of the horizontal faces below $Q_{i}$. The weights $w_{m n}^{(i)}$ were chosen to satisfy $w_{m n}^{(i)} k_{m n}=\ell\left(Q_{i}\right) A_{m_{n} m_{n-1}} / h_{m_{n} m_{n-1}}$, hence

$$
M_{i} u_{i}^{2} \leq L \sum_{(m, n) \in \mathcal{G}_{i}} w_{m n}^{(i)} k_{m n}\left(u_{m}-u_{n}\right)^{2} .
$$

\section{REFERENCES}

[1] I. BABušKa AND A. K. AzIz, On the angle condition in the finite element method, SIAM J. Numer. Anal., 13 (1976), pp. 214-226.

[2] M. Bern, D. Eppstein, And J. R. Gilbert, Provably good mesh generation, in 31st Annual Symposium on Foundations of Computer Science, St. Louis, MO, IEEE Press, Piscataway, NJ, 1990, pp. 231-241.

[3] L. Chew, Guaranteed-Quality Triangular Meshes, Technical Report CS 89-983, Cornell University, Ithaca, NY, 1989.

[4] D. Du And F. Hwang, Computing in Euclidean Geometry, Lecture Notes Ser. Comput. 1, World Scientific, River Edge, NJ, 1992.

[5] R. H. MacNeal, An asymmetrical finite difference network, Quart. Appl. Math., 11 (1953), pp. 295-310.

[6] G. L. Miller, D. Talmor, S. Teng, and N. J. Walkington, A Delaunay based numerical method for three dimensions: Generation, formulation and partition, in Proceedings of the 27th Annual ACM Symposium on Theory of Computing, Las Vegas, NV, ACM Press, NY, 1995.

[7] G. L. Miller, D. Talmor, S. Teng, N. J. Walkington, and H. Wang, Control volume meshes using sphere packing: Generation, refinement and coarsening, in 5th International Meshing Round Table, Pittsburgh, PA, Sandia National Labs, Albuquerque, NM, 1996, pp. $47-62$.

[8] S. A. Mitchell and S. A. Vavasis, Quality mesh generation in three dimensions, in Proceedings of the ACM Computational Geometry Conference, Berlin, Germany, ACM Press, NY, 1992, pp. 212-221 (also appeared as Cornell C.S. TR 92-1267).

[9] R. A. Nicolaides, Direct discretization of planar div-curl problems, SIAM J. Numer. Anal., 29 (1992), pp. 32-56.

[10] J. RuPPERT, A new and simple algorithm for quality 2-dimensional mesh generation, in Third Annual ACM-SIAM Symposium on Discrete Algorithms, Philadelphia, PA, 1992, pp. 8392. 